\title{
Wild type ATTR amyloidosis
}

INSERM

\section{Source}

INSERM. (1999). Orphanet: an online rare disease and orphan drug data base. Wild type ATTR amyloidosis. ORPHA:330001

A rare systemic amyloidosis characterized by combination of various symptoms, depending on the organ involved. Common clinical features are cardiac failure, cardiac conduction anomalies or arrhythmia, renal dysfunction, carpal tunnel syndrome and spinal canal stenosis. Histology reveals fibrillary amyloid deposition of wild type transthyretin mostly in the kidneys, heart, gastrointestinal tract, skin and tenosynovial tissue. 